Cahiers $d u$ MONDE RUSSE

\section{Cahiers du monde russe}

Russie - Empire russe - Union soviétique et États indépendants

$48 / 1 \mid 2007$

Émigrations au début du XXe siècle

\title{
La Pisanelle de Gabriele d'Annunzio et le mirage orientaliste
}

GÉrard Abensour

\section{OpenEdition}

\section{Journals}

Édition électronique

URL : https://journals.openedition.org/monderusse/8986

DOI : 10.4000/monderusse.8986

ISSN : $1777-5388$

Éditeur

Éditions de l'EHESS

\section{Édition imprimée}

Date de publication : 15 janvier 2007

Pagination : 87-108

ISBN : 978-2-7132-2146-0

ISSN : $1252-6576$

Référence électronique

GÉrard Abensour, "La Pisanelle de Gabriele d'Annunzio et le mirage orientaliste », Cahiers du monde russe [En ligne], 48/1 | 2007, mis en ligne le 01 janvier 2007, consulté le 02 septembre 2022. URL : http://journals.openedition.org/monderusse/8986 ; DOI : https://doi.org/10.4000/monderusse.8986 
chercher : repérer : avancer

Cet article est disponible en ligne à l'adresse :

http://www.cairn.info/article.php?ID REVUE=CMR\&ID NUMPUBLIE=CMR 481\&ID ARTICLE=CMR 4810087

\title{
La Pisanelle de Gabriele d'Annunzio et le mirage orientaliste
}

\author{
par GÉrard ABENSOUR
}

\section{Editions de l'EHESS | Cahiers du monde russe}

\author{
$2007 / 1$ - Vol 48 \\ ISSN 1252-6576 | ISBN 9782713221460 | pages 87 à 108
}

Pour citer cet article :

—Abensour G., La Pisanelle de Gabriele d'Annunzio et le mirage orientaliste, Cahiers du monde russe 2007/ 1, Vol 48, p. $87-108$. conditions générales d'utilisation du site ou, le cas échéant, des conditions générales de la licence souscrite par votre établissement. Toute autre reproduction ou représentation, en tout ou partie, sous quelque forme et de quelque manière que ce soit, est interdite sauf accord préalable et écrit de l'éditeur, en dehors des cas prévus par la législation en vigueur en France. Il est précisé que son stockage dans une base de données est également interdit. 


\section{LA PISANELLE DE GABRIELE D'ANNUNZIO ET LE MIRAGE ORIENTALISTE}

Désireux de fuir ses créanciers et ses encombrantes conquêtes, Gabriele d'Annunzio s'installe en 1910 dans une villa d'Arcachon. Cela ne l'empêche pas de faire de nombreux allers et retours à Paris où il va être reçu dans les salons et surtout où il assistera au déferlement de couleurs, de musique et de sensualité qui émane des Ballets russes. En cette année 1910, la troupe de Diaghilev, qui se produit à l'Opéra, séduit par la perfection de son style mais aussi par l'affirmation d'un exotisme exacerbé, qui secoue un public français assez timoré dans ses goûts. Le 4 juin d'Annunzio assiste à la création du ballet Shéhérazade. Il découvre là le talent fulgurant de ceux qui seront bientôt ses complices, Ida Rubinstein pour la danse et Léon Bakst pour le décor et les costumes.

Avec son exubérance latine, l'écrivain italien se serait même, dit-on, agenouillé devant la danseuse, dans sa loge, pour lui baiser les pieds, ou plutôt ses jambes magnifiques. Quant à Bakst, il a été subjugué par les compliments de l'écrivain italien. Témoin cette lettre : «Cher maître et ami, je n'oublierai pas les paroles significatifs (sic) et pénétrantes, que Vous m'avez adressé (sic) sur la scène, pendant Shéhérazade - nul au monde ne me comprendra pas (sic) autant que Vous!»1

Il se crée des liens subtils entre le condottiere et le monde artistique parisien qui, de son côté, se passionne pour la fougue des artistes russes. D’Annunzio est introduit dans les salons par Robert de Montesquiou. Il y rencontre notamment Romaine Brooks, peintre et riche Américaine, qui mettra sa fortune à sa disposition. On doit à cette artiste un très beau portrait en pied de l'écrivain sur fond d'orage. On lui doit aussi une Ida Rubinstein en Vénus triste, un très beau nu qui fait écho en plus

1. Lettre de Léon Bakst à d'Annunzio, du 10 novembre 1910, Archives du Vittoriale. Je tiens à remercier ici tout particulièrement Madame Mariangela Carubini, conservateur du Vittoriale, qui m'a facilité le travail de recherche dans les archives de Gabriele d'Annunzio. 
sensuel à la fameuse toile de Valentin Sérov et qui avait fait scandale en représentant la danseuse nue dans une pose anguleuse.

Nous sommes en 1910. Le thème oriental devient omniprésent. Les Ballets russes ouvrent un nouveau cycle de représentations avec L'Oiseau de feu et les Orientales, deux ballets aux titres parlants ${ }^{2}$. Le public de l'Opéra est ébloui par la splendeur de ces spectacles tout frémissants d'un érotisme sublimé par les recherches esthétiques. Quel enchantement que celui de ces corps souples dansant à la perfection, que la richesse des costumes et des décors, au service d'une musique à la recherche de sonorités nouvelles. Les Ballets russes poursuivent leur entreprise de séduction d'un spectateur français facilement blasé, en colorant ses spectacles d'un érotisme qui trouve sa justification dans l'exotisme oriental. Par sa position géographique et son histoire, la Russie est devenue le vecteur des cultures orientales, turque, persane et caucasienne. Ainsi se révèle un visage de l'Orient très différent de celui que connaissent les Français. Pour la France, l'Orient se situe en Afrique du Nord et au Levant. Les peintres et écrivains, tels Fromentin, Gérôme ou Matisse, vont chercher leur inspiration au Maroc, en Tunisie et en Palestine. Ces civilisations du désert frappent par leur austérité qui tranche avec la sensualité et l'exubérance de l'art persan dont s'inspirent les orientalistes russes.

C'est dans ce contexte que s'inscrit l'apport de d'Annunzio qui va tâcher de marier la clarté latine à la fougue slave. Il est à l'origine de deux réalisations qui font date. Après Le Martyre de Saint-Sébastien où sa plume s'essaye à allier religion et érotisme, d'Annunzio présente en 1913 La Pisanelle ou la mort parfumée qui exploite les ressorts de la vogue orientaliste. Ce mouvement esthétique en plein essor sera malheureusement brisé par les bouleversements en tous genres déclenchés par la Grande Guerre dont les conséquences conduiront à brouiller brutalement tous les repères.

Le ballet Shéhérazade agit comme un choc révélateur sur le dramaturge italien. Sans hésiter, il veut créer une œuvre d'une beauté aussi fulgurante. Il s'adresse aussitôt aux deux principaux artisans de ce succès, le metteur en scène et scénographe du spectacle, Léon Bakst, et la figure hiératique apparue dans le rôle de la favorite Zobéide, Ida Rubinstein.

Le titre de ce court ballet conçu sur la musique de Rimskij-Korsakov se pare du prestige des Mille et une nuits ${ }^{3}$ pour conter une histoire d'amour et de sang dans le cadre évocateur d'un harem. On en rappellera brièvement l'intrigue qui n'en est pas une. Le sultan Chahriyar part à la chasse, laissant sa favorite Zobéide régner sur le

2. Création le 25 juin 1910 de deux ballets dont les vedettes sont Tamara Karsavina et Vaclav Nižinskij : L'Oiseau de feu, variations sur des contes d'Afanas'ev, chorégraphie de Mihail Fokin, musique originale de Stravinskij, décors de Golovin, costumes de Bakst ; Les Orientales, variations sur des danses siamoises, chorégraphie de Mihail Fokin, orchestration par Stravinskij d'œuvres de compositeurs russes (Aleksandr Glazunov, Anton Arenskij et Aleksandr Borodin) et norvégiens (Edouard Grieg et Christina Sinding), décors de Korovin et costumes de Bakst.

3. La nouvelle traduction des Mille et une nuits due au Dr J.-C. Delarue-Maldrus a été publiée en 1904. La femme du traducteur tient un salon réputé qui sera fréquenté par Gabriele d'Annunzio. 
harem. À peine est-il parti que les femmes soudoient le grand eunuque afin qu'il ouvre les portes aux esclaves noirs. Le plus beau d'entre eux (Nižinskij), chamarré d'or, est choisi par Zobéïde (Rubinstein) au cours d'une fête orgiaque. Celle-ci est brutalement interrompue par le retour inopiné du sultan qui ordonne un massacre dont seule réchappe Zobéide. Mais celle-ci se poignarde aux pieds de son maître.

La cruauté et la violence de la scène finale sont accentuées par le contraste entre la panique qui s'empare des victimes cherchant échapper à leur sort et l'immobilité de Zobéide qui observe calmement la scène en attendant avec une majesté tranquille d'accomplir son destin.

D'Annunzio veut écrire une pièce d'une même intensité de passions et prévoit dès l'abord d'en confier la mise en scène à Bakst. Mais Ida Rubinstein, en sa qualité de commanditaire du spectacle, veut réunir autour d'elle tous ceux qui lui ont fait confiance à ses débuts à Saint-Pétersbourg et notamment Vsevolod Meyerhold $^{4}$. Trois hommes, trois artistes, l'avaient aidée à faire ses premiers pas : Bakst, Meyerhold et Fokin. En 1908, elle décide, non sans esprit de provocation, de monter Salomé d'Oscar Wilde. L'auteur anglais sentait le soufre, et cette pièce concentrait tous les traits de l'art décadent honni par la bonne société. Mais cette œuvre était la pierre philosophale du modernisme qui se frayait un chemin partout et aussi en Russie. La grande Komissarževskaja projetait de monter cette pièce dans son théâtre ${ }^{5}$, mais devait se heurter à une interdiction catégorique de la censure. Pour contourner cette interdiction Ida Rubinstein avait décidé de représenter la pièce au cours d'un spectacle de bienfaisance, au théâtre Michel qui abritait la troupe française de Saint-Pétersbourg. La présence de notabilités et de membres de la haute noblesse devait permettre de passer outre aux oukases de la censure. Pour la seconder dans cette entreprise, elle fait appel aux plus grands artistes de la capitale. Glazunov pour la musique de scène, Fokin pour la chorégraphie, Meyerhold pour la mise en scène d'ensemble et Bakst pour les décors et les costumes. (Des esquisses du peintre témoignent d'une vision expressionniste de l'Orient biblique, tranchant avec l'académisme des représentations habituelles de Lieux saints). Mais il était dit que cette tentative serait, elle aussi, arrêtée dans l'œuf. Alertée par le député extrémiste Puriškevič, à la tête de la ligue de l'archange saint Michel, la hiérarchie ecclésiastique en appelle aux autorités civiles. Ida Rubinstein arrivera malgré tout à donner une représentation de la danse des sept voiles dans la salle du Conservatoire. La danseuse s'était fait connaître par toute la société de la capitale. Elle tenait maintenant à réunir autour d'elle tous ceux qui lui avaient fait confiance.

Nous allons donc retrouver au berceau de La Pisanelle les trois bonnes fées que furent Bakst, Meyerhold et Fokin. Cette abondance de talents conduira à une

4. Voir l'article que nous avons consacré à La Pisanelle : Gérard Abensour, « Meyerhold à Paris », Cahiers du Monde russe et soviétique, 5 (1), P., 1964. Sur le même sujet, mais en omettant de citer l'article précédent, Béatrice Picon-Vallin et Alexandros Eifkelidis, « Meyerhol’d stavit Pizanellu », Mnemozina, Artist, Rezisser, Teatr, M., 2004.

5. Mise en scène d'Evreinov et décors de Kalmakov. 
certaine saturation qui se révèlera préjudiciable au succès de la pièce lors de sa création, le 11 juin 1913.

Symbolisme du Nord et symbolisme du Sud se rencontrent chez d'Annunzio qui veut mêler dans son écriture violence occidentale et sensualité orientale. Il écrira donc une pièce haute en couleurs, pleine de brutalité et délicieusement décadente.

Le combat n'était pas gagné d'avance. D'Annunzio décide d'écrire directement en français, langue qu'il possède moins bien qu'il ne le pense. D'autre part, il ne faut pas négliger le fait que l'enthousiasme causé par les Ballets Russes n'est pas partagé par tout le monde. Le célèbre critique de la Revue des deux mondes n'a-t-il pas cette réflexion d'un nationalisme exacerbé : « La lumière qui aujourd'hui nous vient du Nord est une lumière crue, aveuglante et blessante. Disons tout simplement que c'est une invasion de la barbarie. Il est temps qu'elle s'éloigne de nous et reflue vers ses steppes $»^{6}$.

Assez curieusement Debussy, qui vient pourtant de s'associer à d'Annunzio en composant la musique du Martyre de Saint Sébastien, a lui aussi un mouvement de recul. Voici ce qu'il écrit à propos de la nouvelle œuvre du poète, La Pisanelle :

Pourquoi tant occuper les yeux, quand les oreilles ont tout à retenir? Depuis quelques années nous obéissons à des influences où le Nord se concerte avec Byzance pour étouffer notre génie latin, fait de grâce et de clarté. Vous le savez mieux qu'un autre, mon cher ami. Je ne sais pas si ce n'est pas le moment, après l'avoir tant subi, de réagir?

Rien n'arrête d'Annunzio emporté dans son rêve d'une pièce qui se situerait aux marges de l'Occident et de l'Orient. Il emprunte son esthétique au Moyen-Âge et se propose d'écrire un «mystère », revu dans l'esprit du symbolisme. D'où le titre auquel il pense au début, Le Jeu de la rose et de la mort. Non pas « pièce », mais « jeu », ce genre qui est devenu à la mode. Cette allégorie qui met en scène la beauté et la mort rentre bien dans l'esthétique décadente. Le titre définitif sera La Pisanelle ou la mort parfumée. Abandonnant le style naturaliste qui l'avait rendu célèbre, l'auteur de La fille de Jorio adopte ici une langue stylisée et précieuse, pastiche d'ancien français mâtiné d'italien.

Il faudra une musique de scène et c'est au compositeur italien Ildebrando di Parma (Pizetti de son vrai nom) que l'auteur la commande. Il réaliserait, d'après un critique, « un pastiche habile de Shéhérazade, de Thamar et du Prince Igor » ${ }^{8}$. On ne sort pas des Ballets russes, même si musique de ballet et musique de scène ne sont pas interchangeables. Le mariage souhaité entre les passages parlés et la musique ne semble pas avoir été atteint lors de la création de la pièce. Ida Rubinstein en revanche aura tout loisir pour danser sur les rythmes familiers qui lui seront

6. René Doumic, Revue des deux mondes, 15 mai 1913, p. 450.

7. Lettre de Debussy à d'Annunzio du 12 juin 1913. Voir Guy Tosi, Debussy et d'Annunzio, Correspondance inédite, P. : Denoel, 1948.

8. Andrej Levinson, « Pizanella, ili Dušistaja smert’ », Reč`,23 juin 1913. 
proposés. Son rôle est muet et ce sont ses formes splendides et sa danse au dernier acte qui lui assurent les faveurs du public.

Les décors de Bakst sont essentiels dans ce travail. Il a une vision de l'Orient qui vient de loin ${ }^{9}$. Lors de son premier séjour en France, en 1896, le jeune peintre a fréquenté l'atelier du peintre Jean-Léon Gérôme ${ }^{10}$ qui s'est rendu célèbre par ses intérieurs de harem ou de mosquées. Bakst a pu contempler au musée de l'Ermitage des œuvres comme le Bain maure où le peintre joue habilement du contraste entre la peau noire de l'esclave et l'incarnat de sa maîtresse nue dont il lave soigneusement le corps. N'est-ce pas là la préfiguration des scènes de danses lascives de Shéhérazade où se marient la chair blanche des danseuses et la peau sombre des esclaves noirs?

La pièce imaginée par d'Annunzio participe du même monde fait d'oppositions violentes. L'Orient met son empreinte sensuelle sur la chair de La Pisanelle. L'action de la pièce se situe au XII ${ }^{\text {e }}$ siècle, à la fracture de deux mondes et de deux symboliques religieuses. Le schisme entre les Églises d'Occident et d'Orient se profile à l'horizon. On est à Chypre, épicentre des deux mondes. L'obsession n'est pas neuve : d'Annunzio avait proposé en vain à Puccini le livret d'un opéra situé à Chypre. Cette fois-ci, il tient l'équilibre entre l'antique Kypris, l'île d'Aphrodite, nourri de sa forte charge d'érotisme païen et le siège d'une principauté chrétienne. «Dans le royaume latin de Chypre, en l'an de sècheresse où la reine Vénus réapparut près de sa ville d'Amathonte ». Cette préface à la pièce en résume bien le propos ambigu.

Quatre tableaux se succèdent en une rhapsodie pleine de tumulte et de couleurs. Un prologue suivi de trois actes nous conte la légende de la belle esclave de Pise (la Pisanelle), appelée à régner sur l'île de Chypre, et finissant par mourir sous une avalanche de pétales de roses.

1 - Le prologue présente dans une vaste salle de banquet tous les grands du royaume. Les convives attablés discutent ferme. Parmi la centaine de personnages évoqués par l'auteur, on remarque particulièrement «les neuf évêques latins » et «les quatre évêques grecs » qui s'opposent dans une joute théologique. Les titres des convives sonnent comme un feu d'artifices : le prince d'Antioche, le prince de Galilée, le vicomte de Nicosie, le baïle de Venise, le consul pisan, le seigneur d'Arsur, le seigneur de Césarée, le seigneur de Barut, le comte de Paphé, le comte d'Edesse...

9. On remarquera que l'Orient russe est incarné par des sujets juifs de l'Empire russe, à l'intérieur duquel ils sont soumis à un statut discriminatoire, Bakst (né Rosenfeld) provenait de Grodno (Belarus). Il avait embrassé la religion orthodoxe pour épouser la fille du richissime Tret jakov. À la suite de son divorce en 1903 et de son retour à sa religion d'origine, et en vertu des lois de 1'Empire, il n'a plus le droit de résider à Saint-Pétersbourg. Son installation définitive en France en 1909 est la conséquence de cette interdiction. Quant à Ida Rubinstein qui est née à Kharkov (Ukraine), elle doit à l'immense fortune de sa famille le droit de résider dans la capitale en dépit de ses origines.

10. Jean-Léon Gérôme (1825-1904), peintre orientaliste couvert d'honneurs. Il n'aura pas de mots assez durs pour les impressionnistes. 
2 - Le premier acte s'ouvre sur une scène de port. Dans un grouillement de marins, de soldats et de marchands, on voit, au milieu de la cargaison briller « la rose du butin », la Pisanelle, esclave ligotée, à moitié nue, sur le pont du bateau. Elle reçoit les hommages de ses admirateurs, hommages qui s'appliquent également à Ida Rubinstein, titulaire du rôle.

Quand elle marche, elle balance et flatte

ses minces flancs,

ses longues cuisses

et tous nos rêves

sur ses genoux polis

comme jeux d'osselets.

3 - Au deuxième acte, la Pisanelle fuit le désir des hommes en se réfugiant dans une abbaye où les religieuses la vénèrent sous le nom de la « béate » (bienheureuse). Le prince de Tyr, régent du royaume, survient avec sa garde pour enlever cette créature. Il sait que c'est une aventurière qui vit de ses charmes. Mais il est défié par son neveu, le prince Huguet, prétendant au trône, qui est tombé éperdument amoureux de la jeune femme. Dans sa fureur, le prince transperce son oncle de son épée. Il fait venir la Pisanelle à la cour.

4 - Au troisième et dernier acte, la reine mère demande à la Pisanelle de danser pour elle aux sons d'une viole. Elle est décidée à se débarrasser par tous les moyens de l'aventurière qui a enjôlé son fils. Entraînée dans une danse endiablée par trois esclaves numides, la Pisanelle est poussée vers un divan où elles l'étouffent sous un amas de pétales de roses, c'est pour elle la mort parfumée.

Un des protagonistes de cette légende est l'île de Chypre elle-même identifiée à une femme :

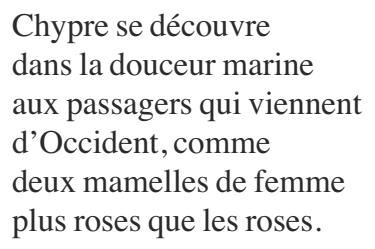

Sur ces terres difficilement conquises sur le paganisme, les chrétiens sont occupés à se disputer, Latins contre Grecs. L'évêque grec d'Amathonte revendique bien haut la supériorité de son église :

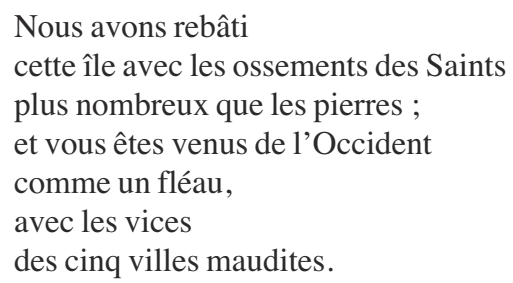




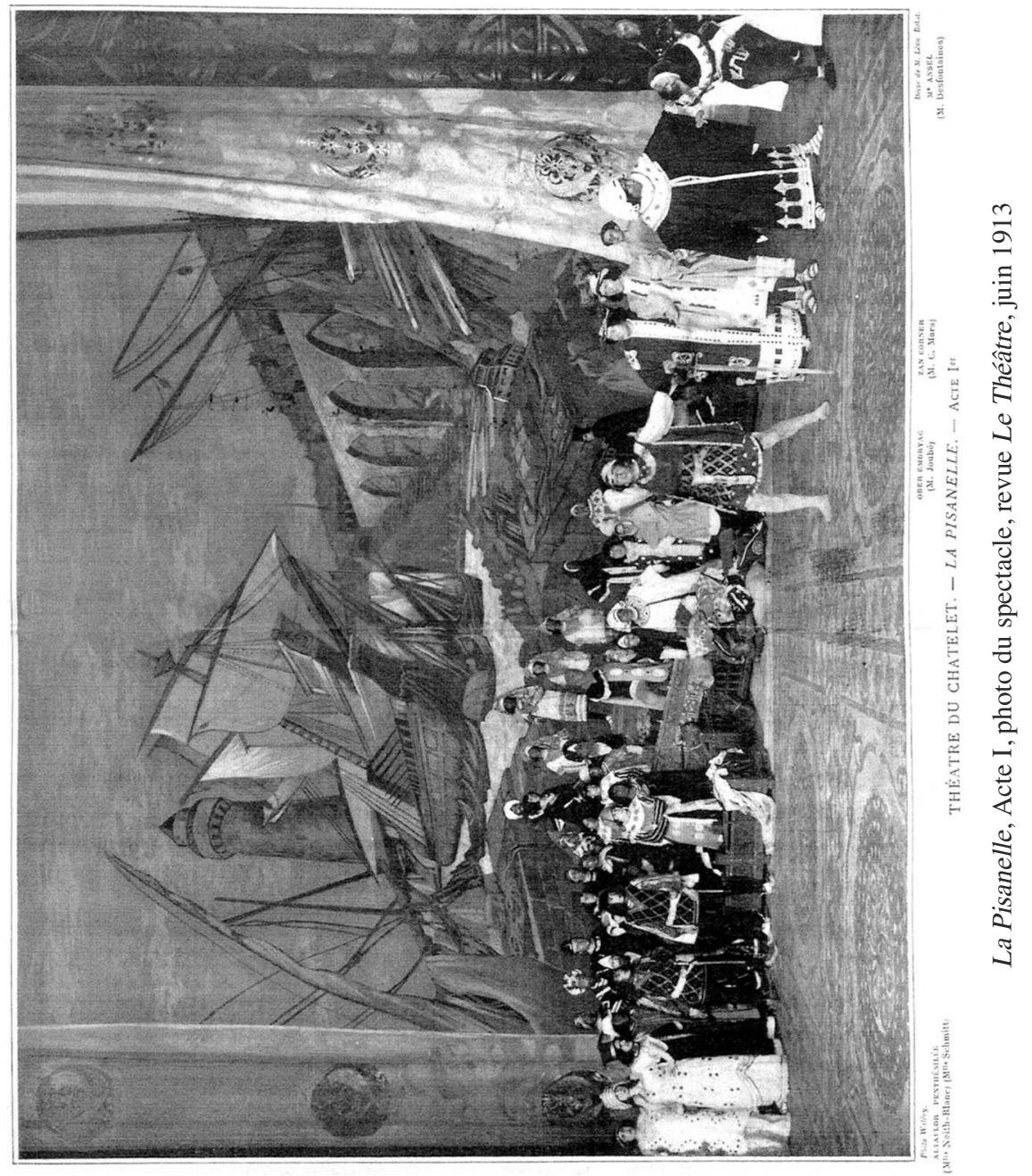


L'évêque latin de Famagouste rétorque avec le même violence :

Les sinagogues et les mahomeries

sont moins infâmes

que vos chapelles...

Comment exprimer avec plus de vigueur l'antagonisme qui s'est installé entre les deux branches du christianisme ? D'Annunzio peut-il espérer par son art réconcilier les Latins et les Orientaux ?

Le personnage de la Pisanelle a été conçu pour Ida Rubinstein dont elle épouse les ambiguïtés. L'actrice a longtemps été dénigrée, surtout dans son pays d'origine, où elle est jalousée pour son immense fortune et où l'on qualifie son art d'amateurisme. Sa biographie parue récemment en France fait litière de ces préjugés ${ }^{11}$. On y découvre une Rubinstein modeste, travailleuse et artiste jusqu'au bout des ongles. Pour se perfectionner, elle prend des leçons de danse avec Fokin et de diction avec Sarah Bernhardt. Elle correspond bien à l'idée que l'on se fait en France de l'orientale. «Par son aspect, par sa nature, Ida Rubinstein représentait sans effort l'héritière de toute la culture orientale $»^{12}$. En l'occurrence, elle sera dans ce spectacle à la fois l'actrice principale et la commanditaire ce qui ne va pas sans difficulté. Elle est aussi la dédicataire de la pièce.

Les rapports entre d'Annunzio et Bakst sont excellents. L'artiste donne le meilleur de lui-même à cette entreprise dont il est un des créateurs. Le peintre se confie en toute franchise à l'écrivain, au point de lui adresser une sorte de confession étonnante :

Je cherche, et j'ai toujours cherché dans la décoration l'expression la plus intense du sentiment de la pièce, que j'entoure d'une ambiance conforme ; j'aime la passion dans la couleur, comme dans la forme, j'adore les belles étoffes, l'or, les diamants et je suis poussé par un désir ardent de rassembler les plus violents contrastes pour obtenir l'impression d'une richesse et d'une passion affolante ! Voilà les éléments que j'adore : la couleur impériale, la belle et sensuelle forme, le sang, l'odeur de la transpiration, la voix étranglée par l'émotion, les spectacles d'émotions profondes, angoissantes et l'orgueil de la belle chair, saine et splendide. ${ }^{13}$

Le langage des couleurs et la force des contrastes seront son motif constant :

J'ai souvent remarqué que chaque couleur avait sa nuance qui exprime parfois la sincérité et la chasteté et parfois la sensualité et même la brutalité, parfois la fierté ou le désespoir. [...] Il y a une nuance de bleu qui peut être la couleur de la Madeleine et une autre qui peut servir de couleur pour Messaline. ${ }^{14}$

11. Jacques Depaulis, Ida Rubinstein, une inconnue jadis célèbre, P. : H. Champion, 1995. Première biographie complète d'Ida Rubinstein.

12. Nozière (Fernand Weyl), critique théâtral au Gil Blas. Depaulis, Ida Rubinstein..., p. 69.

13. Lettre de Léon Bakst à Gabriele d'Annunzio, du 10 novembre 1910, Archives du Vittoriale. On a respecté le style et la typographie de l'épistolier.

14. « Leon Bakst of the Modern Ballet » (interview), New York Tribune, 5 sept. 1915. 
Bakst est enthousiasmé par la pièce que d'Annunzio lui demande de mettre en scène :

Cher et Illustre Maître,

[...] J'aime déjà le personnage de Pisanella car de quelques flèches lumineuses vous l'avez détachée de l'obscurité d'une pièce naissante. Ce qui me charme c'est l'impudicité inouïe - si bien Moyen Âge, si bien ce temps trouble, pieux et magnifique, l'impudicité belle et magistralement cynique de la Pisanella courtisane avide de nouvelles contrées, docte en son métier, duquel elle fait un art, tellement elle sait être sainte et charmeuse..$^{15}$

Retrouvée et publiée par une chercheuse italienne, cette lettre ${ }^{16}$ n'a pas manqué de la surprendre. Elle qualifie de «salaces » les propos du peintre en qui affleure soudain une sensualité qu'il sublimait par son art. Il caractérise ainsi son amie Ida Rubinstein «Si c'est une image de la Kypride, elle garde donc une flamme cachée, une sensualité devinée, sous les traits d'une aventurière ».

Bakst qui se passionne pour la pièce expose ainsi sa vision du deuxième acte :

Qu'il est beau l'acte du monastère ! La fraîcheur matinale, la belle et saine santé de ces fraîches «belettes », essaim affolé, où la beauté et la religion sont délicieusement confondues, l'âme cherchant l'au-delà et le corps adorant et dégustant avec ivresse la beauté terrestre - quelle étrange et nouvelle vision de cette âme enfermée dans un beau corps et cachée sous le scapulaire du Moyen Âge ! [...] Je voudrais que ceci soit joué génialement, sans cliché «grue » ou « chanoinesse » car rarement on a opposé d'une façon si simple et frappante les deux bouts de l'âme «femme »!17

Le trio d'Annunzio, Ida Rubinstein et Bakst se met donc à l'œuvre pour réaliser $L a$ Pisanelle. Sensible aux arguments financiers d'Ida Rubinstein, Fokin de son côté quitte Diaghilev pour régler la chorégraphie de La Pisanelle ${ }^{18}$, tandis que Bakst s'attelle aux décors. Il ne manque plus que Meyerhold. Lorsqu'en 1911, 1'Opéra de Paris avait créé le Martyre de saint Sébastien avec Ida Rubinstein dans le rôle-titre, on s'était peu soucié de la mise en scène. Armand Bour, qui en fut chargé, n'était qu'un amateur. L'artiste russe tenait cette fois-ci à faire appel à un maître incontesté de la mise en scène moderne, Vsevolod Meyerhold.

15. Léon Bakst, lettre à Gabriele d'Annunzio du 28 août 1912, Archives du Vittoriale.

16. Anjuta Maver Lo Gatto, « A proposito di otto lettere di Léon Bakst a Gabriele d'Annunzio e delle messe in scena parigine del Martyre di San Sebastiano e della Pisanella », D'Annunzio nelle culture dei paesi slavi, Venise : Marsilio editore, 1979, p. 68.

17. Lettre de Léon Bakst à Gabriele d'Annunzio du 7 avril 1913, Archives du Vittoriale.

18. Ida Rubinstein décrit à Fokine les deux passages qu'elle souhaite lui voir régler : une courte danse de deux minutes environ au début de la pièce et surtout l'extraordinaire danse de la fin qui conduit à la mort «parfumée » de l'héroïne et qui doit durer sept minutes. Voir l'intéressante étude de N.L. Dunaeva, «Esli vy priedete, to ja spokojna », Zapiski Sankt-peterburgskoj teatral'noj biblioteki, $\mathrm{N}^{\circ} 6-7,2006$, p.29-35. L'auteur a retrouvé dans les archives de la Bibliothèque théâtrale de Saint-Pétersbourg l'original de cette lettre non datée. 


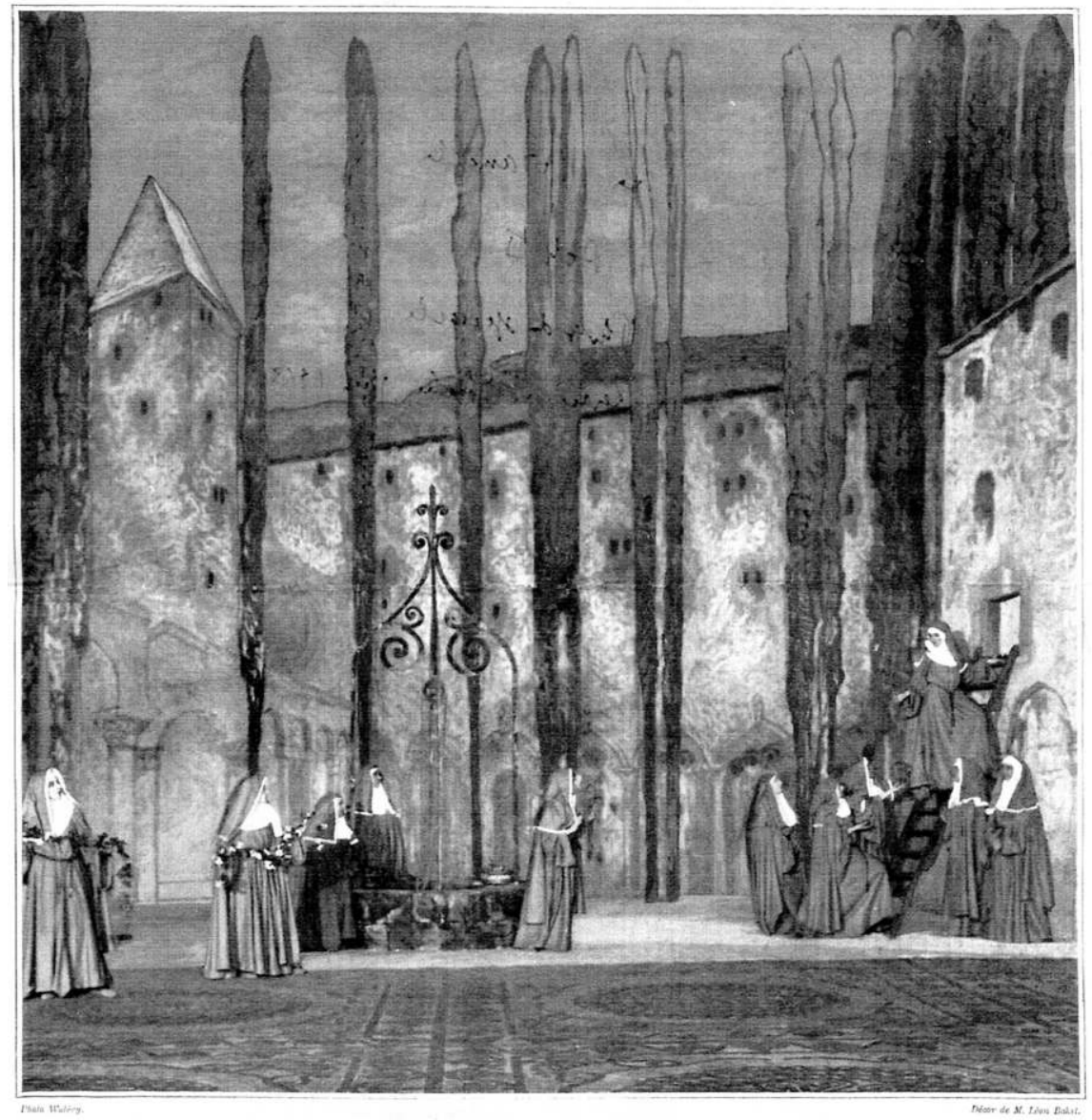

La Pisanelle, Acte II, photo du spectacle, revue Le Théâtre, juin 1913

Se déroule alors une comédie à trois personnages. Ida Rubinstein propose, d'Annunzio se fait prier, tout autant que Meyerhold qui attend une invitation en due forme de l'auteur. Bakst fait, quant à lui, contre mauvaise fortune bon cœur. N'avait-il pas été pressenti pour monter la pièce, comme pour Shéhérazade ? Ce n'est que sur les instances d'Ida Rubinstein, à qui il ne peut rien refuser, qu'il accepte de jouer les intermédiaires. Le prestige de Meyerhold fera le reste.

Ida Rubinstein a sondé Meyerhold dès l'été de l'année précédente. On envisage de monter deux œuvres, celle que promet d'Annunzio et une œuvre de Verhaeren, qui jouit d'une grande estime en Russie. Il s'agit d'Hélène de Sparte qui offre un rôle féminin à la mesure des ambitions d'Ida Rubinstein. L'été 1912 est pour Meyerhold un moment capital de son évolution théâtrale. Il réunit à Terioki, station balnéaire sur le golfe de Finlande à deux pas de Saint-Pétersbourg, des amis comédiens, danseurs et peintres, pour expérimenter des modes nouveaux d'expression 
théâtrale. On se passionne pour la commedia dell'arte, on découvre l'univers tragique de Strindberg.

Il a tant à faire à Saint-Pétersbourg, malgré tout il est séduit par la proposition de l'actrice. Et pourquoi ne pas faire connaître à Paris l'œuvre singulière de Fedor Sologub ? On pourrait avantageusement remplacer Hélène de Sparte par le Don des sages abeilles. Meyerhold voit une parenté certaine entre l'œuvre de Sologub et celle de d'Annunzio. Mêmes débordements de sensualité, même obsession de la mort. Mais la pièce de l'auteur russe devra être traduite en français, ce qui prendrait du temps.

Une correspondance nourrie s'engage alors entre ces trois personnages. Le 11 juillet 1912, Meyerhold écrit de Terioki qu'il lui est impossible de s'engager sans connaître la pièce. Ce sera sa position constante. Il précise sans s'embarrasser de précautions : «J'estime beaucoup les pièces de d'Annunzio sur le plan littéraire mais pas beaucoup sur le plan scénique ${ }^{19}$.

Appelé à Saint-Pétersbourg par l'Académie des beaux-arts, Bakst en profite pour rencontrer Meyerhold. Il lui expose le déroulement de la pièce, mais cela ne peut suffire au metteur en scène qui exige de voir au moins un synopsis détaillé de l'œuvre proposée : «Je dois dire que la pièce m'intéresse. Je vous donnerai ma réponse définitive dès que j'aurai reçu et lu le synopsis ». Il tient à rassurer son interlocutrice et à lui témoigner de son respect : « Ne vous faites pas de soucis. Moi aussi je veux faire quelque chose pour vos sympathiques projets parisiens ${ }^{20}$. C'est pour elle, plus que pour d'Annunzio, qu'il tentera cette aventure.

Quant au dramaturge, il est bien en peine de fournir quoi que ce soit à Meyerhold, car il n'a pas encore écrit un traître mot de sa pièce. Dans une lettre datée du 23 août 1912, Bakst s'enhardit à réclamer de lui au moins une lettre aimable à l'intention de Meyerhold qui est assez susceptible. « J'ai écrit (comme j'ai promis à Mme R) à Meyerhold [...] et je lui ai fait entrevoir une lettre de vous ». Quelques jours plus tard il entre dans le vif du sujet (lettre déjà citée du 28 août 1912) :

[Ce qui me charme] c'est surtout l'atmosphère qui est créée et je la sens vivement - je sens sa particularité, ce que j'ai essayé, peut-être mal, d'en donner une idée à Meyerhold lequel m'écrit une longue lettre où il me prie de Vous demander un aperçu de la pièce dans les grandes lignes - tout de suite car il veut se décider et peut-être sacrifier les mises en scène de Saint-Pétersbourg, afin de s'adonner complètement à Votre œuvre. [...] Si le temps vous permet, écrivez-lui ou envoyez-lui un brouillon de votre main; il donne sa parole d'honneur de garder un secret absolu du milieu, du sujet et de l'époque choisie et, je crois, c'est quelqu'un qui pourra garder un secret. ${ }^{21}$

19. Lettre de Meyerhold à Ida Rubinstein du 11 juillet 1912, Perepiska, M.: Iskusstvo, 1976, p. 144.

20. Lettre de Meyerhold à Ida Rubinstein du 6 octobre 1912, Perepiska, p. 147.

21. Lettre du 28 août 1912, écrite en français, Archives du Vittoriale. Comme on le voit, la syntaxe de Bakst est parfois hésitante. De même pour son orthographe que j'ai en général corrigée. 
Quelques jours plus tard Bakst est à Karslbad d'où il écrit à Meyerhold une lettre qui semble signifier que celui-ci est prêt à s'associer à cette entreprise.

Je viens d'écrire à d'Annunzio. Je vous enverrai son brouillon dans quelques jours sans doute. Faites-moi savoir quand vous l'aurez reçu. Bien sûr je ne touche pas au découpage de la mise en scène de La Pisanelle tant que nous n'en aurons pas parlé. Je travaille sur le caractère des décors, le style ou plutôt les couleurs, les costumes, je recherche une nouvelle ligne pour la silhouette des décors, quant au découpage, nous le réaliserons ensemble, ce n'est qu'ainsi que l'on peut arriver à quelque chose de réussi, n'est-ce pas ?22

Quelques mois plus tard Ida Rubinstein s'inquiète du retard pris par le dramaturge. À Arcachon, où il s'est isolé pour écrire sa pièce, elle lui envoie un télégramme qui traduit son impatience.

Je viens de recevoir une lettre de Meyerhold qui, après une conversation avec Bakst, brûle pour Pisanelle. Il me supplie lui envoyer plus vite scénario il doit maintenant déjà avoir reçu celui que je lui ai envoyé mais je vous supplie de lui envoyer le plus vite possible celui que vous devez faire copier. Ceci je vous assure cher frère est très important. Je vous envoie mes plus tendres pensées. Pisanella ${ }^{23}$.

Meyerhold reçoit enfin le scénario demandé. Quant au texte de la pièce, le dramaturge l'écrit acte après acte, textes qu'il envoie à Paris tout au cours du printemps 1913.

En signe d'acceptation, Meyerhold a envoyé le 26 octobre 1912, un télégramme qu'il signe en compagnie de Bakst :

Admirons scénario Pisanella. Enthousiasmes. Meyerhold Bakst.24

Le même jour, il adresse au maître une lettre dans laquelle il manifeste un respect qui tranche avec sa désinvolture passée. Rubinstein aurait-elle fait la leçon à Meyerhold?

Cher Monsieur,

J'ai reçu votre aimable lettre dans laquelle vous me proposez de monter Votre nouvelle pièce. Ayant pris connaissance du scénario de la Pisanelle je suis séduit par la possibilité de prendre part à la réalisation scénique des beautés qui transparaissent dans votre œuvre. C'est ce qui me pousse à donner mon accord à la proposition du grand poète.

Vsevolod Meyerhold.

22. L. Bakst à Meyerhold, Karlsbad, le 31 août 1912, Perepiska, p. 146 (traduit du russe par moi, GA).

23. Télégramme (en français) d'Ida Rubinstein envoyé le 23 octobre 1912 à d'Annunzio à Arcachon, Archives du Vittoriale.

24. Télégramme envoyé de Saint-Pétersbourg le 26 octobre 1912 par Meyerhold et Bakst à Gabriele d'Annunzio à Arcachon, Archives du Vittoriale. 
Je vous prie de m'excuser pour le retard avec lequel je vous réponds, mais je n'avais pas votre adresse. ${ }^{25}$

À partir de ce moment-là toutes les conditions de la réussite sont réunies. Ida Rubinstein travaille son rôle au fur et à mesure de la réception du manuscrit, tandis que, sans attendre Meyerhold, Bakst dessine les décors et ébauche la mise en scène.

Bakst travaille déjà pour la Pisanelle il est plein d'ardeur. Hier quand je posais pour mon portrait nous n'avons parlé que de notre rêve. Toutes mes pensées sont avec vous. Je travaille et me prépare. Pisanelle. ${ }^{26}$

Elle annonce bientôt l'arrivée prochaine de deux célébrités étrangères,Vsevolod Meyerhold et Ildebrando Pizzetti.

Meyerhold sera à Paris déjà le 11 avril. Il a beaucoup travaillé à sa mise en scène et je crois que vous vous serez content de lui. Il me semble qu'il serait admirable d'avoir les violes sur la scène pour ma première danse. [...] Je suis très heureuse qu'Ildebrando de Parma [Pizzetti] consente à venir à Paris. Je lui ai écrit l'autre jour. Ce serait bien s'il pouvait venir quand vous y serez. Je travaille beaucoup mon rôle et aussi tout le reste. Il faut que cette année, cela soit beau !27

Face aux réalisations picturales détaillées que lui fournit Bakst et qui constituent une sorte de préfiguration de la mise en scène, Meyerhold s'emploie surtout à organiser la profusion des visions colorées du scénographe. Il va s'attacher à conférer une certaine unité aux tableaux disparates qui constituent la pièce. Il doit faire évoluer une masse d'environ cent à cent cinquante acteurs et figurants en évitant désordre et chaos. L'art du maniement des foules qu'il a appris au Théâtre artistique de Moscou, et qu'il a perfectionné dans ses mises en scène d'opéra, lui sera particulièrement utile dans le foisonnement de personnages imaginé par d'Annunzio. Meyerhold commence par modeler les mouvements des personnages principaux, avant de mener de manière quasi militaire la foule des acteurs et figurants qui emplit la scène.

Le résultat a-t-il été à la mesure des efforts entrepris ?

Le Tout-Paris se presse au théâtre du Châtelet pour assister à la première, le mercredi 11 juin 1913. On y trouve surtout des vedettes du monde des lettres (Maurice Barrès, Colette, Serge de Diaghilev, Paul Fort, Henri de Régnier, Maurice Rostand). Il s'agit d'un événement mondain, le public est impressionné par le faste

25. Lettre du 26 octobre 1912 envoyée par Meyerhold à d'Annunzio, Archives du Vittoriale, XLIX, 2, (traduite par moi, GA). Cette lettre a été publiée en russe et en traduction italienne par Cesare de Michelis, «D'Annunzio nella cultura russa » in Giuseppe dell'Agata, Cesare de Michelis, Pietro Marchesani, éds., D’Annunzio nelle culture dei paesi slavi, Venise : Marsilio Editore, 1979, p. 22-23.

26. Télégramme du 17 janvier 1913 d'Ida Rubinstein à Gabriele d'Annunzio, Archives du Vittoriale.

27. Lettre d'Ida Rubinstein, Hôtel Bristol à Paris, à Gabriele d'Annunzio à Arcachon. Datée du 27 mars 1913, Archives du Vittoriale. 
$260 x m u$ sos 1912 .

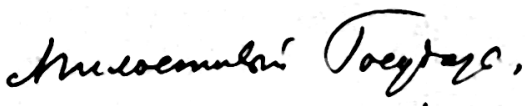

\& nocymen pame uorgave neverco

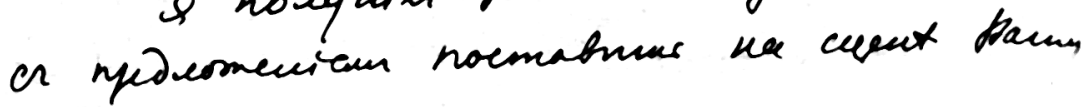
kobyen nteey.

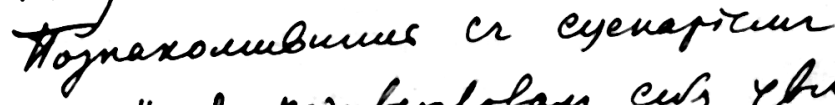
"Tujaceuen", \& nozytemloban cats Yfuerenwhen bojecomevemtio njuntors yracsice $b$

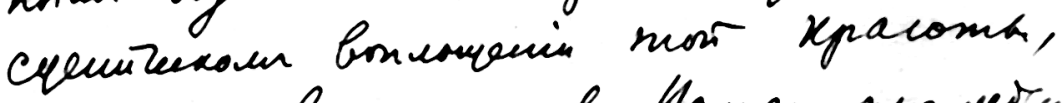

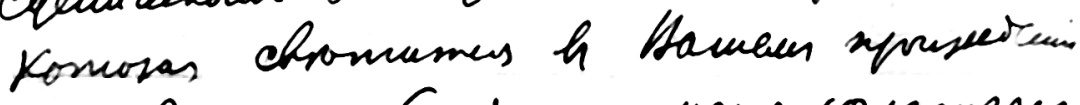

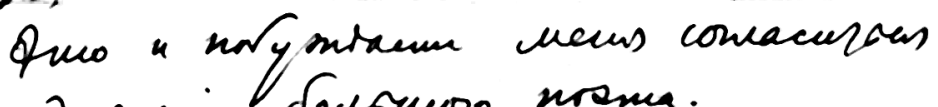

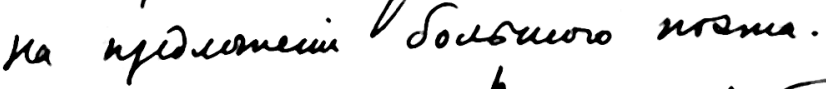
Beboughteriegtaing

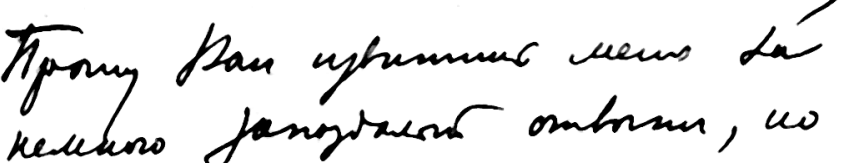
uni kedecoso

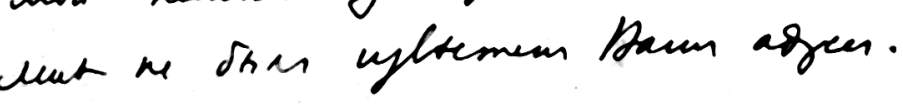

\section{Thempansay nuomatr, 2 , cl. 18.}

Lettre du 26 octobre 1912 envoyée par Meyerhold à d'Annunzio.

Musée d'Annunzio au Vittoriale. Archives XLIX, 2

de la décoration et la notoriété des protagonistes (les acteurs de Max, Suzanne Munte, Hervé). Les critiques français sont en général séduits par le spectacle. On ne peut en dire autant de la presse russe.

La présence de Diaghilev ce soir là est d'autant plus notable qu'il a des raisons d'en vouloir à Ida Rubinstein qui l'a quitté et a entraîné avec elle Mihail Fokin. Remplacé au pied levé par Nižinskij, celui-ci créera deux jours plus tard la chorégraphie du Sacre du printemps, au théâtre des Champs Elysées. Ce sera un événement exceptionnel qui éclipsera, par son archaïsme avant-gardiste, l'orientalisme convenu de La Pisanelle. 
Dans La Pisanelle, les critiques saluent en général la performance d'Ida Rubinstein. On lui sait gré d'avoir perfectionné sa diction tout en continuant à séduire le public par son art du geste et du mouvement.

Son accent a presque tout à fait disparu, et sa voix, d'un beau timbre, a des tonalités d'une séduisante douceur. Sa plastique est superbe. Elle a aussi le don du silence, et ne quitte jamais son personnage. Ses gestes ont de la noblesse, et sa danse est élégante et discrète. ${ }^{28}$

Elle sort du rôle de mannequin charmeur où elle était confinée. Pour la première fois, elle prend la parole. Et c'est à d'Annunzio qu'elle le doit : « D'une danseuse russe, d'Annunzio avait su faire une actrice française. Elle dira plus tard avec une gratitude profonde d'artiste et de femme "Je dansais, je mimais, quand je fis la rencontre de Gabriele d'Annunzio; je puis dire qu'il m'a donné une voix" »29. Les vœux du dramaturge sont comblés.

Le principe adopté par Bakst est celui de l'unité de la palette visuelle, chaque tableau se distinguant par sa coloration particulière : dans le prologue, la couleur dominante est le bleu turquoise ; au 1 ${ }^{\mathrm{er}}$ acte, c'est le rouge ; au 2e acte, le blanc et le bleu ; tandis que le $3^{\mathrm{e}}$ acte est une symphonie de vert. Le scénographe suscite chez certains une admiration sans réserve, mais il rencontre aussi des détracteurs au nom de la simplicité du jeu scénique.

Les décors de M. Bakst révèlent une évolution de ce magicien hardi. Ses architectures sont bien personnelles, mais quelles sonorités, quels flamboiements, quelles audaces heureuses, bien que périlleuses, dans ses oppositions de complémentaires derrière les lambrequins noir et or ! L'atmosphère du second acte est d'un calme presque spirituel. ${ }^{30}$

Mais il y a ceux qu'agacent ses hyperboles et la profusion de couleurs héritée des Ballets russes :

La mise en scène [au sens de la scénographie] a dû coûter fort cher. Pour les costumes et les décors, on n'a rien épargné, c'est certain. J'ai entendu des chœurs d'admiration, auxquels je ne puis mêler ma voix. Les costumes sont nombreux, riches, très recherchés. Ils m'ont paru de couleur trop vive [...] quant aux décors, ils sont de la nouvelle école, et j'avoue préférer l'ancienne. Ce que je prise surtout, dans l'art décoratif, c'est la saillie, la perspective, la reproduction de la nature, et de la vérité, par l'ingéniosité du pinceau. Je suis peut-être un rétrograde, mais je trouve que nous nous méprisons trop facilement. Certes, je ne fais pas fi de l'art russe, malgré ses violences de coloris, mais je préfère encore les délicatesses de l'art français. ${ }^{31}$

28. Félix Duquesnel, « La Quinzaine théâtrale », LeThéâtre, n 348, juin 1913, p. 3.

29. Guy Tosi, «Les relations de d'Annunzio dans le monde du théâtre en France », Quaderni Dannunziani (VI-VII), Vittoriale degli italiani, 1957, p. 57.

30. Michel Georges-Michel, Comoedia, 13 juin 1913.

31. Duquesnel, « La Quinzaine théâtrale », ibid. 


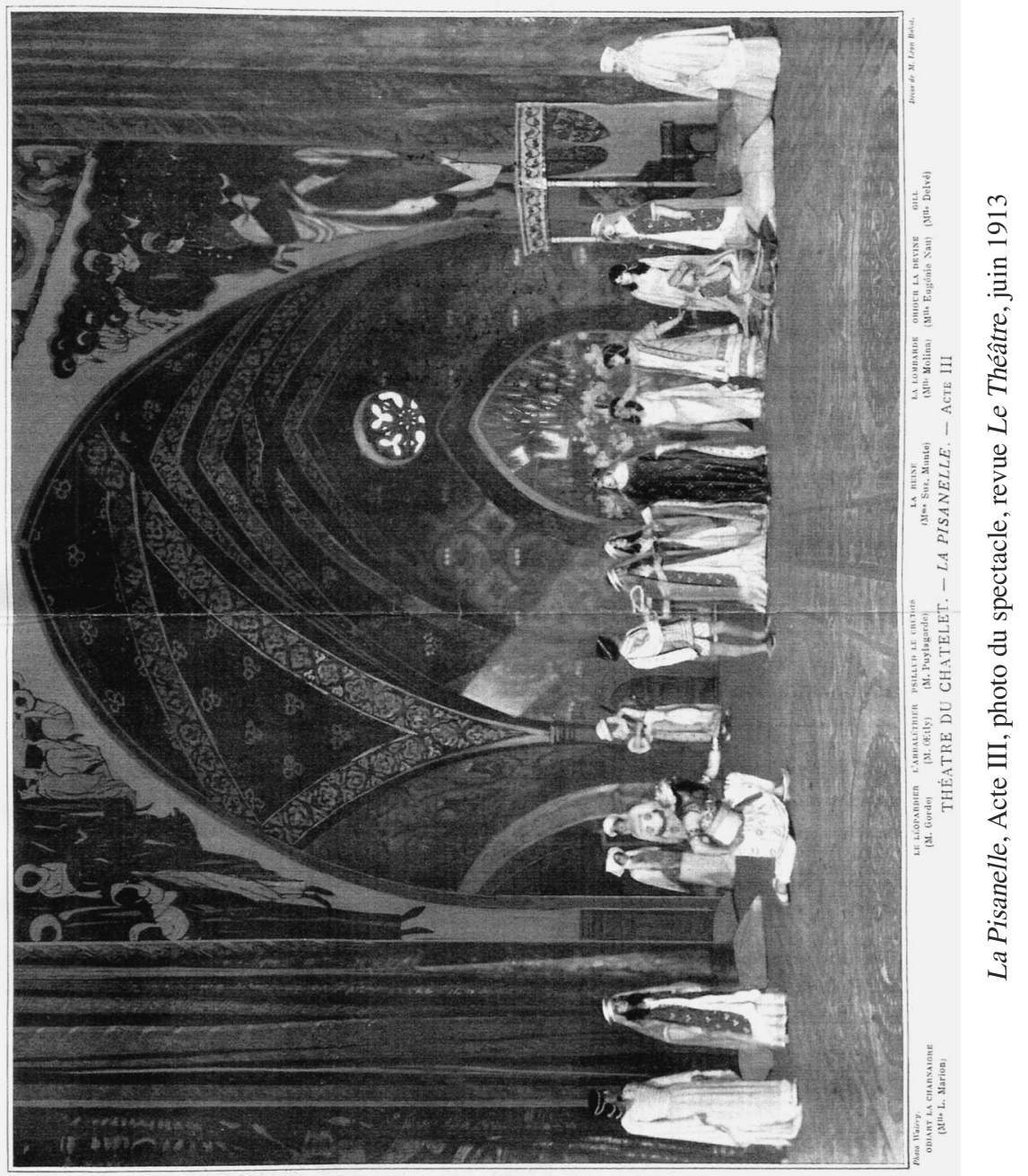


La querelle entre les Anciens et les Modernes reprend ses droits et n'hésite pas à se colorer d'une pointe de xénophobie.

Combien d'étrangers participent à ce spectacle, depuis l'auteur jusqu'au scénographe et à l'actrice principale. À cela s'ajoute la présence de ce metteur en scène russe qui veut imposer sa vision de l'espace scénique à nos habitudes bien françaises !32

Meyerhold avait été prévenu par Bakst des dimensions exceptionnelles du plateau du théâtre du Châtelet, qui n'a de comparable que celui du théâtre Mariinskij, que le metteur en scène russe connaît bien. Du coup, Meyerhold traite la pièce de d'Annunzio comme s'il s'agissait d'un opéra.

La mise en scène a surpris. Presque toute la comédie en larges fresques colorées, se joue au dernier plan. Seules les scènes principales, par un habile retour vers la tradition, se déroulent près du public. Du tumulte, de l'intelligence, du bon goût ; mais un manque de spontanéité qui rend cependant plus décoratif le jeu des artistes. ${ }^{33}$

Gêné par sa méconnaissance du français, Meyerhold ne se rend pas compte du fait que, placés au fond du plateau, les acteurs sont inaudibles. Ou bien ne s'intéresse-til qu'à la cohérence visuelle et musicale du spectacle? Peut-on faire l'économie de l'intelligibilité du texte ? Le critique Duquesne ne peut se contenter d'admirer un beau livre d'images.

Tous les charmes et toutes les finesses du dialogue sont peines perdues. Après quatre heures de spectacle, il ne m'est resté qu'une notion vague. Il m'a fallu deviner ce que je n'entendais pas. Aussi j'ai l'impression d'avoir assisté à un très beau spectacle de mise en scène - encore je ferai tout à l'heure mes réserves -, alors qu'il me faut faire effort pour recomposer le mouvement du drame. [...] Par une imprudence singulière, pour donner plus grand harmonie à la partie décorative, on avait chargé l'avant-scène d'une théorie de rideaux très harmonieux sans doute, dans leurs draperies coloriées et onduleuses, mais qui avaient pour effet d'agrandir encore le proscenium et de forcer les acteurs à jouer au lointain, ce qui rompait toute communication avec le public. ${ }^{34}$

Tout cela n'empêche pas Meyerhold de se délivrer un brevet de satisfaction.

C'est une de mes bonnes mises en scène. Je suis arrivé à unifier les actes qui, chez d'Annunzio manquent tellement d'unité stylistique [...] le premier acte où figurent plus de cent personnes, peut-être deux cents, se déroule avec fluidité et il y a encore beaucoup d'effets que je n'ai pas employés ici [...] Au III acte une

32. Ibid.

33. Georges-Michel, Comoedia, 13 juin 1913.

34. Duquesnel, « La Quinzaine théâtrale », op.cit.p. 2. 
grande nouveauté, l'éloignement par rapport à la rampe, par rapport à la scène avec les figurants en coulisse. Je suis assez content de moi. ${ }^{35}$

En fait on sera obligé de procéder à des aménagements scéniques lors des représentations suivantes. Il y en eut dix au total. Il est vrai que pour Meyerhold, ce spectacle lui était étranger. Il songe plutôt à ses futures activités à Saint-Pétersbourg et en particulier à l'école d'art dramatique qu'il se propose d'ouvrir à l'automne 1913 (le Studio de la rue Trojckaja). Ni dans ses lettres, ni dans ses écrits, il ne parle de La Pisanelle. L'hypothèse la plus probable est qu'il a retrouvé dans l'œuvre du poète italien des échos d'un symbolisme finissant dont il s'éloignait lui-même au même moment.

La pièce de d'Annunzio apparaît comme étouffée sous la profusion des moyens scéniques mis en œuvre. Cette question du rapport entre l'écriture dramatique et sa traduction scénique est un thème récurrent dans la critique française. Henri Ghéon s'interroge à cet égard sur les modifications du statut même du théâtre qui se manifestent dans un spectacle comme celui-ci :

À mesure que se marient plus harmonieusement sur le théâtre, musique, lumière et couleurs, plus déplacé, plus superflu y semble le verbe dramatique. Le spectacle dit tout. A-t-on besoin de mots ? Qu'y a-t-il de trop dans La Pisanelle telle qu'on la représente au Châtelet, sinon la parole rythmée de M. Gabriele d'Annunzio ? [...] Oui, l'art monte sur le théâtre, mais pour nous donner le ballet, la pantomime, la féerie. Et que devient le drame dans tout cela ?36

La question reste posée et sera au centre de l'activité du Cartel qui mènera la lutte contre Sire le mot.

Que pensaient l'un de l'autre d'Annunzio et Meyerhold ? Ils n'en ont rien dit. Ils font connaissance le 24 mai, la seule chose que mentionne Meyerhold, c'est la ressemblance de d'Annunzio avec Mihail Kuzmin, « la féminité en moins ». Quant à l'auteur italien, il fait promettre à Meyerhold de savoir le français la prochaine fois. C'est un conseil que le metteur en scène ne semble pas avoir suivi.

Cette œuvre singulière a-elle marqué les esprits de son impact ? Lunačarskij, le futur commissaire du peuple à la culture, est choqué par le luxe d'un spectacle dont il est de notoriété publique qu'il a été financé par Ida Rubinstein.

Le drame est mis en scène avec un luxe insensé. Il se déroule avec cinq rideaux stylisés encadrés de colonnes gothiques dorées. On aurait pu penser que les combinaisons de losanges, de cercles et de spirales de Bakst et son mélange barbare de couleurs auraient provoqué de la lassitude. Mais de tout l'océan de costumes très riches de la Pisanelle notre peintre a réalisé un tel kaléidoscope que le public, devenu aveugle, a été conquis. Sa cécité n'a pas empêché le public de noter combien le drame et le spectacle étaient conçus pour mettre en valeur

35. Lettre du 20 juin 1903 de Meyerhold à sa femme. Volkov, Mejerxol'd, M.-L. : Academia, 1929 , t. II, p. 291.

36. Henri Ghéon, « Le théâtre du Vieux-Colombier », Revue Bleue, 11 octobre 1913, p. 466. 
celle qui était à l'origine de l'événement et qui jouait le rôle principal, Madame Ida Rubinstein. Comme on le sait, le point faible de cette actrice est la diction et son jeu consiste uniquement en attitudes mystérieuses et muettes. [...] Meyerhold s'agite beaucoup sur la scène et sort de sa manche un truc après l'autre. [...] Je suis sorti de la Pisanelle fatigué par le tourbillon insensé des couleurs, sans compter que j'avais la sensation désagréable d'un spectacle payé terriblement cher, à tous les points de vue, un spectacle qui vous fait tourner la tête sans nourrir l'esprit et qui éveille un sentiment de protestation esthétique, quelque chose qui fait penser à un spectacle de Luna Park. ${ }^{37}$

Tout aussi critique est le point de vue du journal Russkoe slovo.

Ida Rubinstein est une personne affectée, grimaçante et dépourvue d'art. Elle a payé généreusement son droit de figurer dans cette apothéose et de gâcher notre plaisir esthétique. ${ }^{38}$

Monument composite élevé en hommage à l'alliance de l'Orient et de l'Occident, La Pisanelle n'a pas laissé des souvenirs mémorables dans les esprits. Il a cependant été remarqué par un témoin de taille, l'écrivain Ehrenbourg. Celui-ci lui consacre dans ses mémoires quelques lignes ambiguës.

À Paris, Vsevolod Emil'evič se lia d'amitié avec Guillaume Apollinaire, qui apparemment comprit immédiatement que l'important ce n'était ni d'Annunzio, ni Ida Rubinstein, ni les décors de Bakst, mais le désarroi spirituel du jeune metteur en scène pétersbourgeois. ${ }^{39}$

Qu'entendait-il par « désarroi spirituel » ? Dès son retour à Saint-Pétersbourg en juillet 1913 , le « jeune » metteur en scène (il a tout de même près de quarante ans) entrera dans une période d'activité particulièrement faste. Comment peut-on faire confiance à quelqu'un qui avoue n'avoir pas assisté aux faits qu'il décrit ? Il écrit en effet : «En 1913 j'ai raté l'occasion de faire connaissance avec Meyerhold [...] Je savais peu de choses des travaux de Meyerhold, ce que je savais en revanche c'est que d'Annunzio était un phraseur et Ida Rubinstein un dame riche qui aspirait à la gloire théâtrale ${ }^{40}$.

La remarque d'Ehrenbourg est donc à manier avec précaution et témoigne plus des préjugés de son auteur que des sentiments intimes du metteur en scène. Son passage éclair à Paris a-t-il au moins laissé quelque trace dans le monde théâtral français? Selon un des proches de Meyerhold, Aleksandr Fevral'skij, qui partageait notre opinion sur ce point, cette influence s'est surtout exercée dans le domaine de la mise en scène cinématographique, notamment dans le cas d'Abel

37. Anatolij Lunačarskij, « Magdalina i Pizanella », Teatr i Iskusstvo, n²5, 1913, p. 517-518. (traduit du russe par moi, GA).

38. « La Pisanelle à Paris », Russkoe slovo, 12 mai 1913 (a.s).

39. I.G. Erenburg, Ljudi, gody, žizn’, M. : Sovetskij pisatel’, I, 1990, p. 327.

40. Ibid. 
Gance qui avait joué le rôle d'Eudes dans La Pisanelle et qui avait donc pu observer directement l'art du maniement des foules chez Meyerhold. Lorsqu'en 1926 il réalise son premier film à grand spectacle Napoléon, il n'est pas interdit de penser qu'il s'est souvenu des leçons du maître russe ${ }^{41}$.

Cette aventure a marqué de son sceau les protagonistes : Ida Rubinstein signe ses lettres «Pisanelle » et d'Annunzio se prend au jeu. À l'issue d'une visite de l'actrice à l'ermitage de Gardone, visite dont il déplore la brièveté, d'Annunzio lui écrit ce mot plein d'allusions : «La futée Pisanelle s'est sauvée par crainte de la mort parfumée, tandis que mes rosiers dans l'attente devenaient vermeils Stop Elle a très bien agi. J'ai cessé de la rêver et de la chérir. Ariel ${ }^{42}$ ». Leur amitié ne faiblira pas avec le temps et on mesure à leur correspondance la douleur qu'ils ont ressentie à l'annonce de la mort de leur compagnon, Léon Bakst, en décembre 1924. Ida Rubinstein envoie ce télégramme à d'Annunzio :

Notre grand ami Bakst s'est éteint ce matin sans reprendre conscience et sans souffrir Stop Je sais que cette mort vous attristera et me rappelle comme vous m'avez parlé tendrement de lui Stop Je vous embrasse. Ida. ${ }^{43}$

Avec Bakst disparaît la quintessence de l'orientalisme, fondé sur la couleur et la sensualité, qui a séduit une sensibilité occidentale empêtrée dans un rationalisme desséchant. Par un curieux retour des choses, c'est le moment où le constructivisme est en plein essor dans la Russie postrévolutionnaire. L'année de la mort de Bakst est aussi celle où Meyerhold monte La Forêt (Les) d'Ostrovskij et surtout À nous l'Europe (Ehrenbourg, et al.) où il adopte un style anguleux contrastant avec les charmes du «Monde l'art» et des couleurs de l'orientalisme. Le public parisien pourra juger de l'évolution du metteur en scène de La Pisanelle lorsque, lors de sa tournée de 1930, il présentera sa Forêt révisée à l'aune du constructivisme.

Le second séjour de Meyerhold à Paris est à l'origine de certaines hypothèses contestables. On trouve dans une étude sur La Pisanelle la remarque suivante : «L'expérience [de la Pisanelle] lui servira bien plus tard, après le départ de Mihail Čehov, par exemple, lorsque la vie deviendra de plus en plus compliquée et qu'il se posera la question de l'émigration ${ }^{44}$. S'est-il posé la question de «l'émigration»? Certes Jurij Annenkov fait allusion dans ses mémoires au désir de Meyerhold de prolonger la tournée de sa troupe aux États-Unis, reprenant l'expérience de Stanislavskij et du Théâtre artistique. Rien n'indique qu'il songeait à y rester. Si Mihail Čehov, comme l'indique l'article cité, n'est pas retourné en URSS, il est plaisant de constater que Meyerhold l'incite à plusieurs reprises à reprendre du service dans son pays d'origine, offre déclinée à chaque fois par le

41. A. Fevral'skij, Puti k sintezu, M. : Isskustvo, 1978, p. 8. L'auteur fait à ce propos référence à notre article de 1964, (op. cit., note 2, p. 9).

42. Brouillon de télégramme adressé à Ida Rubinstein vers 1925, Archives du Vittoriale.

43. Télégramme d'Ida Rubinstein à Gabriele d'Annunzio, le 28 décembre 1924, Archives du Vittoriale.

44. Béatrice Picon-Vallin et al., op. cit.,p. 494 (retraduit du russe par moi GA). 
LA PISANELLE DE GABRIELE D'ANNUNZIO ET LE MIRAGE ORIENTALISTE

107

Maddme Ida Gubinstein Pace ges ptistollnis, 7 . Foris

Da futé Pisanelle s'est sauvés par orainte de la mort parfumée tandis que mes rosiers Jans l'attente devenaient vermeils. stop. Phe a thes bien agi. J'c cessé de la rêver et de la chérir. Ariel.

Brouillon d'un télégramme adressé à d'Annunzio à Ida Rubinstein (circa 1925). Musée d'Annunzio au Vittoriale. Archives personnelles de d'Annunzio nº 25190

comédien exilé. Celui-ci est très courtisé puisque, rien qu'en 1934, il reçoit de trois directeurs de théâtre des propositions alléchantes : Meyerhold, bien sûr, mais aussi Stanislavskij, au Théâtre artistique et Dal'cev au théâtre Malyj ! Cette offensive de charme n'était pas fortuite.

On mesure combien les bouleversements apportés par la guerre mondiale et ses séquelles ont été fatals à une conception esthétisante du théâtre. Le merveilleux véhiculé par les Ballets russes et, dans son sillage, l'éclairage orientaliste qui avait tant séduit Gabriele d'Annunzio, ne sont plus acceptables dans un monde bouleversé par les horreurs de la guerre mondiale puis de la guerre civile. L'orientalisme est mort, tué par l'Orient. La tentative hardie du poète italien de créer avec La Pisanelle une synthèse colorée des deux composantes de l'esprit européen, l'Orient et l'Occident, n'était plus qu'un mirage. Après la guerre mondiale on assiste au dépeçage de l'Empire turc et à l'émergence d'un empire soviétique qui fait la part belle à ses marches orientales (Congrès des peuples de l'Orient à Bakou en 1920). Entrant de plain-pied dans l'actualité, l'Orient devient un enjeu politique. Quelle place 
reste-t-il pour l' « orientalisme » des contes, au moment où l'Orient sort de sa torpeur pluricentenaire?

Ehrenbourg aurait-il vu juste en qualifiant de non-événement le spectacle fastueux déployé sur la scène du Châtelet en 1913? Lorsqu'il écrit ses mémoires plus de trente ans plus tard, la pulsion universaliste de La Pisanelle a cédé la place au repli nationaliste. Avec sa fougue coutumière, d'Annunzio s'est lancé dans la folle équipée de Fiume qu'il entend rattacher à l'Italie (1919-20) et courtise Mussolini en qui il voit le restaurateur des valeurs nationales. Ida Rubinstein, quant à elle, s'est convertie au catholicisme et découvre la grandeur de Jeanne d'Arc. Elle, persuade Paul Claudel d'écrire une pièce de théâtre dont elle serait l'héroïne ${ }^{45}$ et qui ferait pendant au Martyre de saint Sébastien. Ainsi naît l'oratorio Jeanne au bûcher, dont Honegger compose la musique, et qui devait être créé à l'Opéra de Paris. C'est finalement à Bâle qu'Ida Rubinstein créera le rôle en 1938, avant de le reprendre à Orléans en 1939. Une seconde fois la guerre vient interrompre cette ligne artistique. Ida Rubinstein se réfugie à Vence où elle mourra bientôt. On peut mesurer le chemin qu'elle a parcouru de Kharkov à Orléans, de la courtisane à la sainte, de l'ivresse d'une mort parfumée baignée d'orientalisme à la passion d'une bergère devenue le symbole de la patrie à laquelle Ida Rubinstein a fait don de son talent.

\section{École normale supérieure \\ Lettres et sciences humaines}

gabensour@tiscali.fr

45. Les chercheurs russes ont tendance à minimiser le talent d'Ida Rubinstein et son rôle de catalyseur en matière de modernisme. On est surpris de trouver la concernant des propos injustes et inexacts, comme cette affirmation selon laquelle : « La collaboration avec Paul Claudel n'a rien donné ». (Picon-Vallin et al, op.cit.,p. 613). Ce n'est pas ce qu'écrit le poète : « Arthur Honegger m'avait parlé de la plus remarquable créatrice d'attitudes qui existe actuellement dans le monde, Mme Ida Rubinstein, et que cette artiste me demandait, me commandait une pièce sur Jeanne d'Arc », Paul Claudel, Conferencia, $N^{\circ} X X, 1^{\text {er }}$ octobre 1936. Imaginé par Ida Rubinstein, l'oratorio composé par Claudel et Honegger, s'est révélé être une œuvre majeure du répertoire lyrique du vingtième siècle. 